\title{
GENETIC CONTROL OF TRYPTOPHAN PYRROLASE IN DROSOPHILA MELANOGASTER AND D. VIRILIS
}

\author{
CORRADO BAGLIONI * \\ Institute of Genetics, University of Pavia, Italy $\dagger$
}

Received 23.i.60

\section{INTRODUCTION}

Studies in micro-organisms (Giles, Partridge and Nelson, I957; Yura, I959; Yanofsky and Crawford, I959) have shown that mutant genes can cause synthesis of altered enzymes, either completely inactive or active at a reduced rate (Fincham, 1957). Differences between allelic mutants may result from the production of enzymes altered to different extents (Giles, Partridge and Nelson, 1957; Yanofsky and Crawford, 1959*; Pateman and Fincham, 1958). In some cases such mutant proteins are strongly affected by environmental conditions, as temperature or ion concentration (Horowitz and Fling, I953; Maas and Davis, 1952). Suppressor mutations are useful in discriminating between different alleles, since they can restore the enzymatic activity of some mutants only (Yanofsky and Bonner, I955). In D. melanogaster many allelic series are known, the components of which show different phenotypic traits or different behaviour when coupled with a suppressor gene (for a recent review see Carlson, I959). In this organism it seemed of interest to analyse a case of allelism and the action of a suppressor mutation in terms of the function of the gene product.

Allelism at the vermilion $(v)$ locus $\left(I-4^{\circ} \circ \pm\right)$ was chosen for the analysis. The $v$ locus controls formylkynurenine synthesis from tryptophan and consequently the formation of brown eye pigment, an end product of the tryptophan-kynurenine pathway in insects. When formylkynurenine is added to the food of $v$ larvæ, the eye colour of the adult flies is normal (Green, I952). In a previous paper (Baglioni, I959) it was shown that the enzyme tryptophan pyrrolasef, which catalyzes the oxidation of tryptophan to formylkynurenine, is missing in the alleles $v^{1}$ and $v^{36 f}$. Green (1954) showed that $v^{1}$ and $v^{36 f}$ recombine and interpreted them as pseudoalleles. Moreover the $v^{1}$ allele is suppressed by a suppressor mutation, which does not cause production of brown pigment in flies homozygous for $v^{36 f}$ (Green, 1952). Other alleles, which behave like $v^{1}$, are designated as $v^{s}$ (vermilion suppressible alleles); those behaving like $v^{36 f}$ are designated as $v^{u}$ (vermilion unsuppressible alleles). An additional criterion can be Italy.

* This work was supported by the Comitato Nazionale per le Ricerche Nucleari, Rome,

$\dagger$ Present address : Department of Biology, Massachusetts Institute of Technology, Cambridge, Mass.

$\ddagger$ This enzyme was previously designated as tryptophan peroxidase-oxidase (Knox and Mehler, I950); recently Tanaka and Knox (I959) have pointed out that the enzyme is more accurately described by the term tryptophan pyrrolase. 
used in distinguishing $v^{s}$ from $v^{u}$ alleles ; when larvæ are submitted to partial starvation, at emergence the $v^{s}$ flies show brown pigmentation of the eye, while the $v^{u}$ do not (Green, I954).

For a comparative genetic analysis also two unlinked mutants of $D$. virilis, cardinal $(c d)$ and vermilion ${ }^{48 b}\left(v^{48 b}\right)$ were studied. Both mutants fail to synthesize the brown eye pigment, but become like wild type when supplied with formylkynurenine during larval life (Green, I 952); both accumulate non-protein tryptophan (Green, I949) and are nonautonomous in transplantation experiments, like the $v$ mutants of $D$. melanogaster. The two $D$. virilis mutants can, however, be differentiated by the same tests used with the $v$ alleles of $D$. melanogaster: $c d$ flies show a wild type phenotype in the larval starvation test, while $v^{48 b}$ do not (Green, I954). When $c d$ and $v^{48 b}$ eye discs are transplanted into $D$. melanogaster larvæ with a suppressor (no genes homologous to the $v$ suppressor of $D$. melanogaster are known in $D$. virilis), the $c d$ eye discs become normally pigmented while the $v^{48 b}$ discs do not (Price, I949). Thus $c d$ behaves like the $v^{s}$ alleles of $D$. melanogaster, while $v^{48 b}$ behaves like the $v^{u}$ alleles.

For these reasons Green (1955) suggested that mutants which occur as members of a pseudoallelic system in $D$. melanogaster can occur as unlinked mutants in the $D$. virilis species.

\section{MATERIALS AND METHODS}

Strains : mutant strains of D. melanogaster $v^{1}, v^{36 f}, s u^{2}-s v^{1}, s u^{s 2}-p r-v, s u^{3}-s c v v f$ and $s u^{2}-s w a c v t$ were obtained from the California Institute of Technology; the strains $s u^{2}-s v^{1}$; $b w$ and $s u^{2}-s v^{36 f}$; $b w$ were kindly supplied by Dr M. M. Green. Mutant strains of $D$. virilis $v^{480}$, $c d$ and es were obtained from the Department of Zoology, University of Chicago. The wild type strains of $D$. melanogaster and $D$. virilis and all the mutant strains of $D$. melanogaster not mentioned above, were from the species collection of our Institute.

Enzyme preparation: flies were bred on a standard corn-meal-agar medium at $25^{\circ}$; flies isolated within a day after emergence were transferred into vials with fresh medium, where they were aged three to five days before being used. After being aged the flies were collected and immediately used for assay of tryptophan pyrrolase activity. Crude enzyme extracts were prepared by homogenising the flies in four volumes by weight of a solution $0.14 \mathrm{M}$ in KCl and $0.0025 \mathrm{M}$ in NaOH. After centrifugation ( $20 \mathrm{~min}$. at $\mathrm{I} 6, \mathrm{ooo}$ g.) the clear supernatants were used for enzyme assay.

Enzyme assay: incubation mixtures contained $\mathrm{I} \mathrm{ml}$. of $0.2 \mathrm{M}$ phosphate buffer $p \mathrm{H}_{7}, 0.3 \mathrm{ml}$. of L-tryptophan $0.3 \mathrm{M}$ and $\mathrm{I}$ or $2 \mathrm{ml}$. of enzyme extracts (final volume $3.3 \mathrm{ml}$.). The samples were incubated for 3 hours at $37^{\circ}$ in a Brown shaker and the reaction was stopped by the addition of $\mathrm{I} \cdot \mathrm{I} \mathrm{ml}$. of 20 per cent. trichloroacetic acid. After filtration the kynurenine produced was determined by the Bratton-Marshall reaction, as described previously (Baglioni, 1959). Duplicate determinations were carried out for each experiment and the activities calculated by difference with blanks in which tryptophan was omitted.

Mutants of D. melanogaster were tested in combination with brown (bw) ; homogenates from these strains give lower blank values, because the red eye pigment, the synthesis of which is prevented by the gene bw, absorbs at $560 \mathrm{~m} \mu$ (the wavelength at which the diazotised kynurenine was read). The mutant $c d$ of $D$. virilis was tested in combination with eosinoid (es), which prevents in this species the formation of the 
red pigment. Since Glassman (1956) has shown that kynurenine formamidase, which catalyses the hydrolysis of formylkynurenine to kynurenine, is present in normal amount in all the strains tested, it was assumed that formylkynurenine formed from tryptophan was quantitatively transformed into kynurenine.

Special medium: feeding experiments were performed by adding tryptophan to the medium, 5 per cent. brewers' yeast and $I \cdot 5$ per cent. agar. Vials were autoclaved before use and L-tryptophan added, before the medium had solidified, to a final concentration of 0.25 per cent. 20-30 eggs rinsed in ethanol and wiped with filter paper were transferred in each vial.

\section{TRYPTOPHAN PYRROLASE ACTIVITY OF D. melanogaster STRAINS}

Tryptophan pyrrolase activity was determined in two wild type strains of $D$. melanogaster and in mutant strairs of this species, in which the synthesis of the brown pigment was blocked at different steps of the tryptophan-ommochrome pathway. The results are reported in table I. The results of a previous investigation (Baglioni, r959),

TABLE 1

Tryptophan pyrrolase activity of wild type and mutant strains of Drosophila melanogaster

\begin{tabular}{|c|c|c|}
\hline Strain & Mean activity \pm standard error & No. of experiments \\
\hline 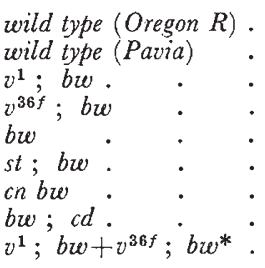 & $\begin{array}{l}133 \pm 11 \\
82 \pm 12 \\
14 \pm 2 \\
10 \pm 2 \\
138 \pm 15 \\
235 \pm 25 \\
192 \pm 22 \\
110 \pm 11 \\
9 \pm 2\end{array}$ & $\begin{array}{l}6 \\
3 \\
6 \\
5 \\
3 \\
6 \\
6 \\
3 \\
3\end{array}$ \\
\hline
\end{tabular}

The tryptophan pyrrolase activity is expressed as $\mu$ moles $\times 10^{-3}$ of kynurenine formed by one gram of flies in one hour at $37^{\circ}$.

* In these experiments equal amounts of the extracts of the two strains were mixed before the incubation.

concerned with the tryptophan pyrrolase activity of the pseudoalleles $v^{1}$ and $v^{36 f}$ are also reported for comparison.

The tryptophan pyrrolase activity of the Oregon $R$ wild type flies is greater than that of the Pavia wild type strain; this is probably due to genetic differences between the two lines. The mutants cinnabar $(c n)$ and scarlet $(s t)$ displayed a considerably greater enzyme activity than the wild type. The concentration of the enzyme may perhaps increase in mutants which block tryptophan metabolism; the evidence is not conclusive, since the strains examined have different genetic backgrounds. In this connection it is important to point out that the tryptophan pyrrolase activity of cardinal $(c d)$ flies, in which brown pigment formation is incompletely blocked but strongly lowered, is quite normal.

When extracts of $v^{1} ; b w$ and $v^{36 f} ; b w$ were incubated together, no increase in activity was observed. It seems therefore unlikely that the $v$ pseudoalleles act on two successive steps of a chain of reactions. 


\section{THE ACTION OF THE SUPPRESSORS OF VERMILION}

The suppressors of vermilion ( $\mathrm{I}-\mathrm{O} \cdot \mathrm{O}+$ ) are assumed to be members of an allelic series; these suppressor genes have no visible effect when alone and can be recognised only when combined with the mutant genes they suppress. Three suppressor strains were tested for tryptophan pyrrolase activity in combination with the suppressible mutants $v^{1}: s u^{2}-s, s u^{3}-s$ and $s u^{s}-v-p r$. The suppressor $s u^{2}-s$ was also tested in combination with $v^{1}$; bw, with $v^{36 f}$; bw and not combined with a $v$ mutant in the strain $s u^{2}-s w w^{a} c v t$.

The results of tryptophan pyrrolase determination in suppressor strains are reported in table 2.

A partial recovery of enzyme activity takes place in the presence of

TABLE 2

Tryptophan pyrrolase activity of suppressor of $\mathrm{v}$ strains

\begin{tabular}{|c|c|c|}
\hline Strain & Mean activity \pm standard error & No. of experiments \\
\hline $\begin{array}{l}s u^{2}-s v^{1} \cdot \\
s u^{2}-s v^{1} ; b w \\
s u^{2}-s v^{36 f} ; b w . \\
s u^{3}-s c v v^{1} f \quad \cdot \\
s S^{2}-v-p r v^{1}: \\
s u^{2}-s w^{a} c v t \\
s u^{2}-s v^{1}+v^{1} ; \quad b w^{*} \\
s u^{2}-s v^{1}+v^{36 f} ; b w^{*}\end{array}$ & $\begin{array}{r}26 \pm 6 \\
26 \pm 3 \\
8 \pm 1 \\
22 \pm 3 \\
19 \pm 4 \\
103 \pm 9 \\
24 \pm 3 \\
18 \pm 2\end{array}$ & $\begin{array}{l}6 \\
9 \\
3 \\
6 \\
6 \\
5 \\
3 \\
3\end{array}$ \\
\hline
\end{tabular}

The tryptophan pyrrolase activity is expressed as $\mu$ moles $\times 10^{-3}$ of kynurenine formed by one gram of flies in one hour at $37^{\circ}$.

* In these experiments equal a mounts of the extracts of the two strains were mixed before the incubation.

suppressor genes; in the unsuppressed strain $s u^{2}-s v^{36 f}$; bw a tryptophan pyrrolase activity greater than that of the $v^{36 f}$; bw strain was not observed.

When enzyme extracts of strain $s u^{2}-s$ were mixed and incubated together with those of the $v^{1} ; b w$ and $v^{36 f} ; b w$ strains, no increase of tryptophan pyrrolase activity beyond the activities determined separately was observed. Thus the action of the suppressor genes seems to be limited to the restoration of the tryptophan pyrrolase of the $v^{1}$ mutant in vivo.

The action of the suppressor genes seems to be qualitatively identical; they increase the rate of tryptophan oxidation in the $v^{1}$ strain but not in the $v^{36 f}$. The quantitative significance of the results was examined by a $t$ test (see table 3 ). The action of the suppressor genes on the tryptophan pyrrolase activity of the $v^{1}$ mutant is small, although significant in at least two of the three strains examined.

The tryptophan pyrrolase activity of the strain $s u^{2}-s w w^{a} c v t$ seems to be like that of the wild type ; the suppressor genes apparently have no effect on the enzyme activity of the $v^{+}$allele.

The introduction of the mutation cinnabar $(c n)$ in the strain $s u^{2}-s v^{1}$; 
$b w$ gave further evidence that the suppressors of $v$ are concerned with the activation of tryptophan pyrrolase in the $v^{1}$ mutant and do not provide an alternate pathway of tryptophan metabolism. The mutant $c n$ is known to lack the ability to oxidise kynurenine to $3-\mathrm{OH}-$ kynurenine. Crossing $c n$ bw flies and $s u^{2}-s v^{1}$ flies to a lethal balanced strain and crossing the progeny in the proper way, the strain $s u^{2}-s v^{1}$; $c n$ bw was selected. The $s u^{2}-s v^{1}$; $c n$ bw flies are white eyed. Since

TABLE 3

Statistical test of the significance of the differences between the mean tryptophan pyrrolase activity of the strain $\mathrm{v}^{1}$; bw and the mean activities of the suppressed $\mathrm{v}^{1}$ strains

\begin{tabular}{|c|c|c|}
\hline Strain & $t$ values & Probability range \\
\hline$s u^{2}-s v^{1} ; b w$. & $3 \cdot 245$ & $0.0 \mathrm{I}-0 \cdot 00 \mathrm{I}$ \\
$s u^{3}-s c v v^{1} f$. & $2 \cdot 280$ & $0.05-0.02$ \\
$s u s^{2}-v-p r v^{1}$ & $\mathrm{I} \cdot \mathrm{II} 9$ & $0.2-0.3$ \\
\hline
\end{tabular}

the phenotypic manifestation of the suppressor gene is hidden by the mutation $\mathrm{cn}$, it can be concluded that the suppressor of $v$ does not provide a way to bypass the metabolic block of the $c n$ mutants and that its action is limited to the immediately preceding metabolic step, namely the oxidation of tryptophan to kynurenine.

Attempts have also been made to determine the action of the

TABLE $_{4}$

\begin{tabular}{|c|c|c|}
\hline Cross & $\begin{array}{l}\text { Genotype of the } \\
\text { female progeny }\end{array}$ & Phenotype \\
\hline$s u^{2}-s v^{1} ; b w \times v^{1} ; b w$ & $\frac{s t^{2}-s}{+} v^{1} ; b w$ & Diluted brownish \\
\hline$s u^{2}-s v^{1} ; b w \times s u^{2}-s v^{36 f} ; b w$ & $s u^{2}-s \frac{v^{1}}{v^{36 f}} ; b w$ & $\begin{array}{c}\text { Brown } \\
\left.\text { (lighter than } s u^{2}-s v^{1} ; b w\right)\end{array}$ \\
\hline
\end{tabular}

suppressor gene $s u^{2}-s$ on heterozygous $v^{1} / v^{36 f}$ females and of the heterozygous suppressor gene on $v^{1}$; bw flies.

Proper crosses were done and the phenotype of the female progeny examined for eye pigmentation; the results are reported in table 4 . The heterozygous $s u^{2}-s \frac{v^{1}}{v^{36 f}} ; b w$ females are suppressed to an intermediate level. Their pigmentation is intermediate between that of $s u^{2}-s v^{1} ; b w$ flies and that of $v^{1} ; b w$. This fact supports the idea that the two pseudoallelic genes $v^{1}$ and $v^{36 f}$ work quite independently from each other and that the suppression is due only to the amount of the product of the $v^{1}$ gene present. 
The heterozygous suppressor has a relatively weak effect on the pigmentation of $v^{1}$; bw flies ; it is not, however, a true recessive since heterozygous $\frac{s u^{2}-s}{+} v^{1} ; b w$ females can be easily distinguished from $v^{1} ; b w$ flies.

\section{DIFFERENTIATION OF VERMILION PSEUDOALLELES}

Valadares and Jacquot (I952) observed a slight pigmentation in $v$ flies when the larvæ were grown in a sterile medium supplemented with L-tryptophan. We were able to show that the mutant $v^{1}$ produces some brown pigment when fed tryptophan, while $v^{36 f}$ does not. These mutants were tested in combination with brown (bw). The larvæ were supplied with a special medium, in which tryptophan was present at a concentration of 0.25 per cent. (see Materials and Methods).

The pigmentation of the tryptophan-fed $v^{1} ; b w$ flies was clearly appreciable, although the quantity of the pigment formed was much less than in wild type flies. The identity of the pigment produced by the tryptophan-fed $v^{1} ; b w$ flies with the brown pigment of the wild type was investigated with a chromatographic test. The flies were prepared for chromatography according to the methods of Hadorn and Mitchell (r95I) and the chromatograms were then developed in the solvent collidine/o.5 $\mathrm{M} \mathrm{KH}_{2}{ }^{\mathrm{P}} \mathrm{O}_{4}$ (2/3). A spot identified as xanthommatin, according to Butenandt and Neubert (1955), was observed in chromatograms obtained from wild type and in smaller amount in the chromatograms from tryptophan-fed $v^{1}$; bw flies.

\section{TRYPTOPHAN PYRROLASE ACTIVITY OF D. VIRILIS STRAINS}

The results of tryptophan pyrrolase determinations in $D$. virilis strains are shown in table 5. The tryptophan pyrrolase activity of the

TABLE 5

Tryptophan pyrrolase activity of $\mathrm{D}$. virilis strains

\begin{tabular}{|c|c|c|}
\hline Strain & Mean activity \pm standard error & No. of experiments \\
\hline $\begin{array}{l}\text { wild type. } \\
v^{48 \mathrm{~b}} . \\
c d . \\
c d ; \text { es } \\
\text { wild type }+v^{48 \mathrm{~b} *} \\
\text { wild type+cd*. } \\
c d+v^{48 \mathrm{~b} *} .\end{array}$ & $\begin{array}{l}86 \pm 6 \\
6 \pm 0 \cdot 5 \\
49 \pm 3 \\
84 \pm 4 \\
47 \pm 3 \\
63 \pm 3 \\
26 \pm 2\end{array}$ & $\begin{array}{l}8 \\
6 \\
8 \\
8 \\
3 \\
3 \\
3 \\
3\end{array}$ \\
\hline
\end{tabular}

The tryptophan pyrrolase activity is expressed as $\mu$ moles $\times 10^{-3}$ of kynurenine formed by one gram of flies in one hour at $37^{\circ}$.

* In these experiments equal amounts of the extracts of the two strains were mixed before the incubation.

$v^{48 b}$ flies is reduced to a very low level ; the activity of the $c d$ mutant is only slightly lower than that of wild type, or as in the case of the $c d$; es flies, equal to that of the wild type. 
When enzyme extracts of the strains examined were mixed before incubation, no increase nor inhibition of the tryptophan pyrrolase activity was recorded. In all cases the activity corresponded, within the limits of random fluctuations, to the mean of the activities determined separately.

\section{DISCUSSION}

The incubation with L-tryptophan of extracts of mutant strains $v^{1} ; b w$ and $v^{36 f}$; bw yields less than I/ I o of the amount of diazotisable amine produced by the wild type. The specificity of the BrattonMarshall reaction being relatively low, it cannot be established whether the small amount of diazotisable material formed by the $v$ pseudoalleles is kynurenine or not.

No spot corresponding to kynurenine appears on paper chromatograms of the concentrated incubation mixtures (Baglioni, I959). But this does not rule out that the diazotisable material may be kynurenine, since this test is not sensitive enough. Alternatively the diazotisable material may be some other aromatic amine produced from tryptophan by the extracts.

It is indeed impossible to establish if $v^{1}$ and $v^{36 f}$ flies are completely devoid of tryptophan pyrrolase activity, although it seems likely that these mutant strains lack this enzyme.

The present data exclude the possibility that the two $v$ alleles examined are concerned with successive steps in a sequence of reactions, as suggested for some $D$. melanogaster alleles by the Lewis ( 1956 ) hypothesis of the physiology of pseudoallelism. Our results are instead consistent with the interpretation of pseudoallelism proposed by Pontecorvo ( I 956) on the basis of studies on white alleles of $D$. melanogaster and on biotin mutants of Aspergillus nidulans. According to Pontecorvo (1956) pseudoalleles are mutated at sites, separable by crossing-over, of a large physiological gene. They are therefore truly alleles.

Gene $v$ seems to consist of only one cistron, since complementation between alleles has never been observed. The mutations $v^{1}$ and $v^{36} f \mathrm{im}$ plicate changes at sites, which are a few recombination units apart from one another, but a single physiological function, namely the production of the enzyme tryptophan pyrrolase, is affected. The difference in the behaviour of $v$ alleles shown by Green's ( 1952 ; I 954) results and by the present results, are to be ascribed to the alteration of the gene product induced by the mutation. The mutant $v^{36 f}$ forms a greatly altered gene product, inactive in conditions which allow a partial recovery of the mutant $v^{1}$. It is quite probable that the action of the suppressors of $v^{1}$ lead to the activation of an enzyme less severely altered than that of the $v^{36 f}$ alleles. The suppressor genes increase slightly the tryptophan pyrrolase activity of $v^{1}$, but the effect of the suppressor genes might be more marked during the pupal life, when the brown pigment is more actively synthetised. The low activity of the 
extracts makes it hard to investigate the tryptophan pyrrolase activity at various developmental stages.

The hypothesis that the suppressors of $v$ act by activating an inactive enzyme is supported by the following considerations :-

(i) The non-specificity of suppressor genes, which like the suppressor of $v$ suppress several unrelated mutant genes, could be better explained by supposing that the suppressor modifies an environmental factor, which prevents the function of the mutated genes, than by claiming complicated metabolic relationships. The suppressors of $v$ are known to suppress beside the $v^{s}$ alleles the mutant sable, speck and purple. According to Pontecorvo (I958) " the suppressor acts by modifying some cell conditions to which the changed products of several genes are all sensitive, though only those changed in particular ways."

(ii) The partial suppression of $v^{s}$ mutants by treatments such as larval starvation or tryptophan administration proves that the enzyme of these mutants can function when environmental conditions are changed in particular ways.

(iii) The heterozygous $s u^{2}-s \frac{v^{1}}{v^{36 f}}$; $b w$ flies synthesize a much higher quantity of brown pigment than the heterozygous $v^{1} \frac{s u^{2}-s}{+}$; bw flies. Thus the modification induced by the homozygous suppressor seems to be the most important condition for the suppression, while the dose of the $v^{1}$ gene present determines a quantitative response. The loci $v$ of $D$. melanogaster and $D$. virilis, which are located in homologous segments of the $X$-chromosome, have an identical function since they are both concerned with the synthesis of tryptophan pyrrolase. The mutant $v^{48 b}$ is lacking this enzyme; it cannot be established if it is completely devoid of tryptophan oxidising ability. Considerations similar to those reported above for the $v$ pseudoalleles are valid also for the mutant $v^{48 b}$ of $D$. virilis.

The gene $c d$ of $D$. virilis inhibits brown pigment formation by a quite different mechanism; the brown pigment is indeed present in the ommatidia of $c d$ flies in reduced amount. No explanation can be advanced so far as to the action of the $c d$ gene.

\section{SUMMARY}

I. The tryptophan pyrrolase activity of two wild type strains of $D$. melanogaster and of several mutant strains was determined. Only the allelic mutants $v^{1}$ and $v^{36 f}$ were found to have an activity considerably lower than that of the wild type.

2. The suppressors of $v s u^{2}-s, s u^{3}-s$ and $s u^{S 2_{-}}$were found to increase the tryptophan pyrrolase activity of the $v^{1}$ mutant but not of the $v^{36 f}$ mutant. Further evidence was provided that the action of the suppressors of $v$ is concerned with the metabolic step leading from tryptophan to kynurenine and does not provide an alternate metabolic pathway. 
3. The two alleles $v^{1}$ and $v^{36 f}$ can be differentiated if supplied during larval life with a medium containing high concentrations of L-tryptophan. In these conditions the mutant $v^{1}$ synthesizes brown pigment, while the $v^{36 f}$ does not.

4. The mutant $v^{48 b}$ of $D$. virilis was found to lack the enzyme tryptophan pyrrolase; the mutant $c d$, which is likewise devoid of brown pigment and responds to formylkynurenine administration, showed a normal tryptophan pyrrolase activity.

5. The results are discussed in view of the theories on pseudoallelism of Lewis (1956) and Pontecorvo (1956). There is a one locus-one enzyme relationship in the case of the loci $v$ of $D$. melanogaster and $D$. virilis. The action of the suppressors of $v$ is discussed and the conclusion is reached that these genes activate alternate gene products by changing some cell condition.

Acknowledgments.-I am greatly indebted to Prof. A. Buzzati-Traverso for continual advice, encouragement and helpful discussion throughout the course of this work and to Dr A. Guglielmi for skilful assistance. My thanks are due to Prof. S. E. Luria, to Prof. M. M. Green, to Dr W. E. Knox and to Dr H. W. Lewis for reading this paper and for several improvements and corrections.

\section{REFERENCES}

BAGLIONI, c. 1959. Genetic control of tryptophan peroxidase-oxidase in Drosophila melanogaster. Nature, 184, 1084-1085.

BUtenand, A., AND Neubert, G. 1955. Über Ommochrome. V: Xanthommatin, ein Augenfarbstoff der Schmeissfliege Calliphora eritrocephala. Hoppe Seyler's Z. Physiol. Chem., 3or, Io9-1 I 4 .

CARLSON, E. A. I959. Comparative genetics of complex loci. Quart. Rev. Biol., $34,33-67$.

FinchAM, J. R. S. 1957. A modified glutamic acid dehydrogenase as a result of gene mutation in Neurospora crassa. Biochem. 7., 65, $721-728$.

Giles, N. H., PARTRIDGE, G. W. H., AND NELSON, N. J. I 957 . The genetic control of adenylosuccinase in Neurospora crassa. P.N.A.S., 43, 305-31 7 .

Glassman, E. 1956. Kynurenine formamidase in mutants of Drosophila. Genetics, 4 , $566-574$.

GREEN, M. M. I949. A study of tryptophane in eye colour mutants of Drosophila. Genetics, 34, 564-572.

GREen, M. M. I $95^{2}$. Mutant isoalleles at the vermilion locus in Drosophila melanogaster. P.N.A.S., 38, 300-305.

GReEN, м. м. 1954. Pseudo-allelism at the vermilion locus in Drosophila melanogaster. P.N.A.S., 40, 92-99.

GreEn, m. m. i955. Pseudoallelism and the gene concept. Am. Nat., 89, 65-7 I.

hadorn, H., AND mitchell, H. K. I95I. Properties of mutants of Drosophila melanogaster and changes during development as revealed by paper chromatography. P.N.A.S., 37, 650-665.

HOROWITZ, N. H., AND FLING, M. 1953. Genetic determination of tyrosinase thermostability in Neurospora. Genetics, 38, 360-374.

KNOX, W. E., AND MEHLER, A. H. I95o. The conversion of tryptophan to kynurenine in liver. I. The coupled tryptophan peroxidase-oxidase system forming formylkynurenine. F. Biol. Chem., I87, 419-430.

LEWIS, E. B. 1955. Some aspects of position pseudoallelism. Am. Nat., 89, 73-89.

MAAS, W. K., AND DAVIS, B. D. I952. Production of an altered pantothenatesynthesizing enzyme by a temperature-sensitive mutant of Escherichia coli. P.N.A.S., 38, 785-797. 
PATEMAN, J. A., AND FINCHAM, J. R. S. 1958. Gene-enzyme relationships at the am locus in Neurospora crassa. Heredity, I2, 31 7-332.

Ponteconvo, G. 1956. Allelism. Cold Spring Harbor Symp. Quant. Biol., I6, I 7 II83.

PONTECoRvo, G. 1958. Trends in genetical analysis, Columbia University Press, New York, p. $5^{0 .}$

PRICE, J. B. I949. Transplantation experiments in Drosophila virilis : the formation of brown pigment. Univ. Texas Publ., 4920, 24-30.

SUSKIND, S. R., AND KUREK, L. I. I959. On a mechanism of suppressor gene regulation of tryptophan synthetase activity in Neurospora crassa. P.N.A.S., 45, I97-204.

TANAKA, T., AND KNOX, W. E. I959. The nature and mechanism of the tryptophan pyrrolase (peroxidase-oxidase) reaction of Pseudomonas and of rat liver. $\mathcal{F}$. Biol. Chem., 234, I $162-1$ I 70.

VALADARES-DA-COSTA, M., AND JACQUOT, R. 1952. Effets d'une surcharge en tryptophane sur le development et la coloration de l'oeil des mutants $v$ et $v b w$ de Drosophila melanogaster en fonction du taux protidique du milieu. Compt. Rend., 234, I214-1216.

YANOFSKY, C., AND BONNER, D. M. I955. Gene interaction in tryptophan synthetase formation. Genetics, 40, 761-769.

YANOFSKY, C., AND CRAWFORD, I. P. I959. The effects of deletions, point mutations, reversions and suppressor mutations on the two components of the tryptophan synthetase of Escherichia coli. P.N.A.S., 45, 1о 16-1026.

yURA, T. I959. Genetic alteration of pyrroline-5-carboxilate reductase in Neurospora crassa. P.N.A.S., 45, 197-204. 